\title{
SYMMETRIC CONTINUITY OF REAL FUNCTIONS
}

\author{
C. L. BELNA
}

\begin{abstract}
It is shown that the set of points where a real function is both symmetrically continuous and not continuous has inner measure zero but may have full outer measure
\end{abstract}

We shall consider an arbitrary function $f$ that maps the real line $\mathbf{R}$ into itself. Such a function is said to be symmetrically continuous at $x \in \mathbf{R}$ if

$$
f(x+h)-f(x-h) \rightarrow 0 \text { as } h \rightarrow 0 .
$$

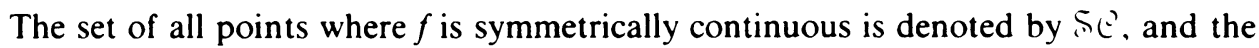
set of all points where $f$ is discontinuous is denoted by $\mathcal{Q}$. (Recall that $\mathscr{D}$ is of type $F_{\sigma}$; on the other hand, $S \subseteq$ need not be measurable and need not possess the Baire property-examples are given below.)

The object of this investigation is the set $s \mathcal{E} \cap$ Q 2 . Interest in this set apparently dates back to 1935 when F. Hausdorff [3] asked whether $(1)$ could be uncountable when $\delta E=\mathbf{R}$; an affirmative answer to this question was given in 1971 by D. Preiss [4]. Hausdorff also asked whether an arbitrarily prescribed $F_{\sigma}$-set could equal of when $\delta \mathcal{E}=\mathbf{R}$. In response to this question, H. Fried [2] proved the following result.

THEOREM $\mathrm{F}$. If $\mathcal{S} \mathcal{C}$ is residual, then $\mathscr{D}$ is of first category.

The analogue for $f$ restricted to an interval is clearly valid; and because $\mathscr{D}$ has the Baire property and any residual subset of an open interval has the Baire property, this theorem has the following equivalent formulations.

THEOREM $\mathrm{F}^{*}$. $S \circlearrowright \cap \mathcal{D}$ is residual on no open interval; or, equivalently, $\delta \in \cap$ OD contains no set of second category that has the Baire property.

In the following sense, Theorem $\mathrm{F}^{*}$ is the "best" statement that can be made concerning the category of $\delta \subset \cap \mathscr{D}$. P. Erdös [1] has shown that the continuum hypothesis implies the existence of an additive subgroup $G$ of $\mathbf{R}$ that is of measure 0 and of second category on every open interval. Thus, if $f$ is the characteristic function of $G$, then $G \subset \mathcal{S} \mathcal{C}$ and $\mathscr{D}=\mathbf{R}$; that is, $\mathcal{\complement} \cap \mathscr{D}$ is of second category on every open interval.

Erdös also proved that the continuum hypothesis implies the existence of a subgroup $G$ of $\mathbf{R}$ that is of first category and has full exterior measure on each open

Received by the editors March 12, 1982.

1980 Mathematics Subject Classification. Primary 26A15. 
interval. Thus, if $f$ is the characteristic function of $G$, then

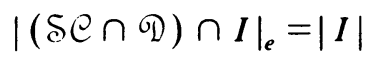

for each open interval $I$ (where $|\cdot|_{e}$ and $|\cdot|$ denote exterior Lebesgue measure and Lebesgue measure). Consequently, the following metric analogue of Theorem $\mathrm{F}^{*}$ is

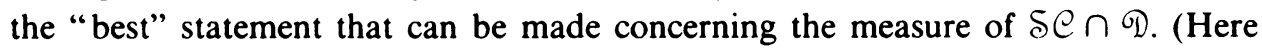
$|\cdot|_{i}$ denotes inner Lebesgue measure.)

THEOREM. $|S \odot \cap \mathscr{D}|_{i}=0$.

It is our goal to furnish a proof of this theorem. First we note an immediate

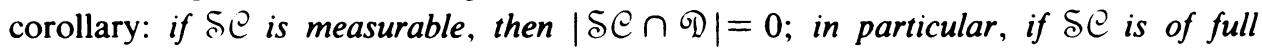
measure, then $|\mathcal{D}|=0$. This contains a previous result of Preiss [4], who proved that $S \mathcal{C}=\mathbf{R}$ implies $\left.\right|^{\mathscr{D}} \mid=0$. We also note that E. M. Stein and A. Zygmund [5, Lemma 9] presented our theorem with the added hypothesis that $f$ be measurable. However, their proof contains an oversight; for example, if $f(x)=x$, then in their notation $E=\mathbf{R}$ and $E_{n}=\varnothing$ for each $n$. (In a footnote [5, p. 266], they do allude to a second proof which may be correct.)

To begin the proof, we introduce an auxiliary function of two variables and prove two key lemmas concerning it. For each $x \in \mathbf{R}$ and each $\varepsilon>0$ we define $\delta(x ; \varepsilon)$ to be the supremum of all numbers $\delta \geqslant 0$ for which

$$
|f(x+h)-f(x-h)|<\varepsilon \quad \text { whenever }|h| \leqslant \delta .
$$

(Note that $\delta(x ; \varepsilon) \geqslant 0$, and that $\delta(x ; \varepsilon)>0$ for each $\varepsilon>0$ if and only if $x \in \mathcal{S}$.)

Lemma 1. Let $\varepsilon$ and $\delta$ be positive numbers, and let $I$ be an open interval with $|I|<\delta / 2$. If $|\{x: \delta(x ; \varepsilon)>\delta\} \cap I|_{e}>0$, then there exists a positive number $\tau$ such that $\delta(x ; 5 \varepsilon)>\tau$ for each $x \in \mathcal{S} \cap \cap$.

Proof. Set $E=\{x: \delta(x ; \varepsilon)>\delta\} \cap I$. Since $|E|_{e}>0$, the distance set

$$
d(E) \equiv\{|x-y|: x, y \in E\}
$$

is dense on some interval $(0, \tilde{\tau})$. (This follows from the identity $\overline{d(E)}=d(\bar{E})$ and the well-known fact that the distance set of a set of positive measure contains some interval $(0, \tilde{\tau})$.) Choose any point $x \in \mathcal{S} \cap I$, and without loss of generality assume $x=0$. Let $h \in(0, \tilde{\tau})$ be arbitrary. To establish the lemma for any $\tau \in(0, \tilde{\tau})$, we shall show that

$$
|f(h)-f(-h)|<5 \varepsilon .
$$

Inequality (1) is certainly satisfied if $\delta(0 ; \varepsilon)>h$. Suppose $\delta(0 ; \varepsilon) \leqslant h$. Because $d(E)$ is dense on $(0, \tilde{\tau})$, there exist two points $x, y \in E$ such that $x<y$ that

$$
0<h-2(y-x)<\delta(0 ; \varepsilon) \text {. }
$$

Let $h_{y}$ be the reflection of $h$ in $y$, and let $h_{y x}$ be the reflection of $h_{y}$ in $x$. Since $\tau \leqslant|I|<\delta / 2$, it readily follows that

$$
|h-y|<|I|+\tau<\delta \text { and }\left|h_{y}-x\right|<|I|+\tau<\delta .
$$


Thus, since $x, y \in E$ we have

$$
\left|f(h)-f\left(h_{y}\right)\right|<\varepsilon \text { and }\left|f\left(h_{y}\right)-f\left(h_{y x}\right)\right|<\varepsilon
$$

that is,

$$
\left|f(h)-f\left(h_{y x}\right)\right|<2 \varepsilon .
$$

A similar argument shows that

$$
\left|f(-h)-f\left((-h)_{x y}\right)\right|<2 \varepsilon
$$

where $(-h)_{x y}$ is the reflection in $y$ of the reflection of $-h$ in $x$. Furthermore,

$$
(-h)_{x y}=-h+2(y-x)=-h_{y x},
$$

and by (2) we have $0<h_{y x}<\delta(0 ; \varepsilon)$; therefore,

$$
\left|f\left(h_{y x}\right)-f\left((-h)_{x y}\right)\right|<\varepsilon .
$$

This together with (3) and (4) and the triangle inequality gives (1), and the proof is complete.

LEMMA 2. Let $\varepsilon$ and $\delta$ be positive numbers, and let $I$ be an open interval with $|I|<\delta$. If $|\{x: \delta(x ; \varepsilon)>\delta\} \cap I|_{i}>3|I| / 4$, then $|f(a)-f(b)|<2 \varepsilon$ for any two points $a, b \in I$.

Proof. If $E=\{x: \delta(x ; \varepsilon)>\delta\} \cap I$, then it readily follows from the hypothesis on $E$ that $(0,|I| / 2) \subset d(E)$. Thus if $a, b \in I$ with $a<b$, there exist points $x, y \in E$ such that $x<y$ and $b-a=2(y-x)$. Let $a_{x}$ denote the reflection of $a$ in $x$, and let $a_{x y}$ denote the reflection of $a_{x}$ in $y$. Because $|I|<\delta$ and $a_{x y}=b$ we have $|a-x|<\delta$ and $\left|a_{x}-y\right|<\delta$; and because $x, y \in E$ we have

$$
\left|f(a)-f\left(a_{x}\right)\right|<\varepsilon \text { and }\left|f\left(a_{x}\right)-f\left(a_{x y}\right)\right|<\varepsilon .
$$

As $a_{x y}=b$, this implies that $|f(a)-f(b)|<2 \varepsilon$, and the lemma is established.

Proof of Theorem. Assume $|\delta \circlearrowright \cap \mathscr{D}|_{i}>0$. If $\mathscr{D}_{t}$ denotes the set of points where the oscillation of $f$ exceeds $t$, then $\left|S \subset \cap \mathscr{D}_{t}\right|_{i}>0$ for some $t>0$ (since each $\mathscr{D}_{t}$ is measurable). Let $M$ be a measurable subset of $\mathcal{S} \cap \mathcal{D}_{t}$ with $|M|>0$. Choose any

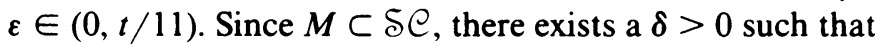

$$
|\{x: \delta(x, \varepsilon)>\delta\} \cap M|_{e}>0 .
$$

Therefore, there exists a point $p \in\{x: \delta(x ; \varepsilon)>\delta\} \cap M$ that is a point of density of $M$ and a point of exterior density of the set $\{x: \delta(x ; \varepsilon)>\delta\}$. Let $I$ be an open interval containing $p$ with $|I|<\delta / 2$. By Lemma 1 there exists a $\tau>0$ such that $\delta(x ; 5 \varepsilon)>\tau$ for each $x \in M$. Let $J$ be an open interval containing $p$ such that $|J|<\tau$ and $|M \cap J|>3|J| / 4$. Then

$$
|\{x: \delta(x ; 5 \varepsilon)>\tau\} \cap J|_{i}>3|J| / 4,
$$

and by Lemma 2 the oscillation of $f$ on $J$ does not exceed $10 \varepsilon$ which is less than $10 t / 11$. This contradicts $p \in \mathscr{D}_{t}$, and the theorem is proved. 
The author wishes to thank M. J. Evans for bringing reference [5] to his attention.

\section{REFERENCES}

1. P. Erdös, Some remarks on subgroups of real numbers, Colloq. Math. 42 (1979), 119-120.

2. H. Fried, Über die symmetrische Stetigkeit von Funktionen, Fund. Math. 29 (1937), 134-137.

3. F. Hausdorff, Probleme \#62, Fund. Math. 25 (1935), 578.

4. D. Preiss, A note on symmetrically continuous functions, Časopis Pěst. Mat. 96 (1971), 262-264.

5. E. M. Stein and A. Zygmund, On the differentiability of functions, Studia Math. 23 (1964), 247-283.

Syracuse Research Corporation, Merrill LaNe, Syracuse, New York 13210 